\title{
Apple of Sodom (Calatropis procera) Callus Extract, a Novel Skincare Active and Its Biological Activity in Skin Models When Combined with Dead Sea Water
}

\author{
Meital Portugal-Cohen 1,2*, Eliran Ish-Shalom ${ }^{1,2}$, Ruben Mallon ${ }^{3}$, Patricia Corral3, \\ Franck Michoux ${ }^{3}$, Ze'evi Ma'or ${ }^{1,2}$ \\ ${ }^{1}$ Ahava Dead Sea Laboratories, Lod, Israel \\ ${ }^{2}$ The Dead Sea Laboratory for Skin Biochemistry and Biotechnology, Dead Sea and \\ Arava Science Center, Masada, Israel \\ ${ }^{3}$ Evonik Advanced Botanicals, Parçay Meslay, France \\ Email:*meital.p@ahava.com
}

How to cite this paper: Portugal-Cohen, M., Ish-Shalom, E., Mallon, R., Corral, P., Michoux, F. and Ma'or, Z. (2018) Apple of Sodom (Calatropis procera) Callus Extract, a Novel Skincare Active and Its Biological Activity in Skin Models When Combined with Dead Sea Water. Journal of Cosmetics, Dermatological Sciences and Applications, 8, 73-91.

https://doi.org/10.4236/jcdsa.2018.82010

Received: March 22, 2018

Accepted: June 26, 2018

Published: June 29, 2018

Copyright $\odot 2018$ by authors and Scientific Research Publishing Inc. This work is licensed under the Creative Commons Attribution International License (CC BY 4.0).

http://creativecommons.org/licenses/by/4.0/

\section{(c) (i) Open Access}

\begin{abstract}
Background: Calotropis procera ( $C$. procera), is an authentic plant naturally grown in the flora of Dead Sea region. Despite its toxicity, $C$. procera presents healing properties. However, it has not been implemented yet in cosmetics as an active ingredient. Objective: The biological effects of $C$. procera callus extract on skin were elucidated solely and in combination with Dead Sea water (DSW). Methods: The capability of $C$. procera extract to protect against skin inflammation and irritation was tested on ex vivo human skin organ culture by LPS and SDS addition respectively. Viability and cytokine secretion were evaluated. The combination of $C$. procera extract with Dead Sea water was tested on full thickness skin equivalents. Gene expression and relevant biochemical markers for glycolysis, hypoxia and extracellular matrix balance were tested. Results: $C$. procera extract exhibits a protective biological activity against skin irritation and inflammation at the biochemical level. Furthermore, a combination of $C$. procera extract and DSW demonstrates a potential contribution for skin wellbeing via enhance energy production, resistance to hypoxia and extracellular matrix balance. Conclusions: Topical application of C. procera callus extract might support skin balance and wellbeing at the molecular level. Hence, it is recommended for new cosmetic formulae as standalone or in combination with Dead Sea water, in the effort to achieve anti-aging bio-activity that is working beyond skin aging symptoms, especially via skin calming effects and skin energy enhancement.
\end{abstract}




\section{Keywords}

C. procera Callus Extract, Alternative Skin Models, Inflammation, Irritation, Aging, Dead Sea Water

\section{Introduction}

Authenticity is an increasing trend in last years' beauty business, changing popular list of ingredients to include more endemic ingredients in new cosmetic launches, especially selective minerals collected from specific lands, local springs, spas and seas, and extracts of endemic plants [1]. The growing awareness to side effects of synthetic drugs invigorates scientists exploring ethnic medicinal remedies as potential new actives for dermatological and cosmetic formulae.

The plant Calotropis procera, also known as "Apple of Sodom" is grown as a woody shrub in desert oases in the hot regions and is a native plant in the flora of Dead Sea region [2] [3]. The extracts derived from C. procera's different plant parts are believed to possess various powerful deeds including anti-bacterial, anti-inflammatory, anti-diabetic and anti-cancer [2] [3] [4]. In spite of its poisoning threats, various skin problems, such as wounds, sores, external infections, swelling and eczema, are treated by $C$. procera's preparations "prescribed" by traditional healers [2] [3]. Yet, $C$. procera has not been exploited yet as an active to be formulated in modern skincare products.

The benefits of Dead Sea minerals for skin health and beauty are well established and formulating Dead Sea different extracts, i.e. DS Mud, DS solid Salts and DS brines, are widespread in modern cosmetics [5]-[10]. Blending Dead Sea minerals and plant extracts were proven to have skin beneficial effects and different mineral-plant extract combinations are formulated and patented as innovative complexes for new cosmetic products [9] [11] [12]. Due to regulatory banning of animal tests [13], skin models are effectively used today, looking for the bio mechanism beyond natural and pathogenic skin phenomena. The wish to successfully attenuate skin aging is the main engine of huge beauty industry [14]. Skin aging reflects the accumulation of many damaged molecules during skin exposure to a broad spectrum of stressors [15]. This inevitable accumulation leads to the activation of "pro-aging" biochemical pathways and could eventually result with the appearance of skin aging symptoms and skin pathologies, dermatitis, rosacea, seborrheic and other pathological skin conditions including skin cancers [16] [17] [18]. Inflammatory processes are key mediators of the different pathways leading to skin aging and therefore, many research activities are targeting protective effects against inflammation using modern skin laboratory in vitro and ex vivo models [19].

This work describes the results of a research, focused on the biological anti-aging and anti-inflammatory skin effects of a callus extract of the plant $C$. procera when topically applied and combined with Dead Sea water. 


\section{Methods}

\subsection{Preparation of Test Materials}

Test materials consist of either C. procera callus extract, either Dead Sea water (DSW) extract (Osmoter), or their combination.

\subsection{Calotropis procera Extract}

Calotropis procera callus was developed from root explants grown under aseptic conditions on Murashige and Skoog medium [20] supplemented with 3\% sucrose, $27 \mu \mathrm{M}$ naphthalene acetic acid, $0.5 \mu \mathrm{M}$ Thidiazuron and solidified with $0.25 \%$ gelrite.

Calotropis procera dried callus was grounded and callus powder was extracted with hot water by using a Soxhlet extractor during $3 \mathrm{~h}$. After extraction, the solvent was partially evaporated, using a rotary evaporator (RC600, KNF). The concentrated solvent with the crude extract was frozen at $-80^{\circ} \mathrm{C}$ for $24 \mathrm{~h}$ and freeze-dried until complete dryness. Crude extract was ground and solubilized in vegetable glycerol and water at a final ratio of $4 \%$ crude extract, $16 \%$ water and $80 \%$ glycerol.

Chemical analysis by HPLC and mass spectroscopy of Calotropis procera callus extract: The dry extract was re-suspended in $250 \mu \mathrm{L}$ of $80 \% \mathrm{MeOH}$. Prior to injection $(20 \mu \mathrm{l})$ into the HPLC system, the sample was filtered through a 0.45 $\mu \mathrm{m}$ filter (Minisart RC4, Sartorius). Analysis of the presence of cardenolides was performed by an HPLC Jasco System instrument consisting of a pump (Pu-2089 Plus) and a diode array detector (MD 2018 Plus). The HPLC column was Luna 2 C18 $(250 \times 4.6 \mathrm{~mm}, 5 \mu \mathrm{m})$ fitted with a guard cartridge (Securityguard system, Phenomenex, France). A linear gradient at a flow of $0.7 \mathrm{ml} / \mathrm{min}$ was used for 25 min. The mobile phase consisted of a solvent A (ultra pure water) and a solvent B (pure Acetonitrile). Gradient was as follow: 0 - 4 min 25\% solvent B; $24 \mathrm{~min}$ solvent B increased to $50 \% ; 29$ min solvent B increased to $70 \%$ and $31 \mathrm{~min} 100 \%$ solvent B. All solvents used were of HPLC grade quality. Detection wavelength was $220 \mathrm{~nm}$. Mass identification of major peaks was performed with an UPLC-ESI/MS consisted of a LC system Acquity I-Class UPLC with a PDA detector linked to a mass spectrometry Instrument Waters LCT Premier (Milford, MA, USA). The chromatographic separation was realized with a C18 UPLC Column $(50 \times 2.1 \mathrm{~mm}, 1.7 \mu \mathrm{m})$. The mobile phase consisted of A: water $+0.9 \%$ formic acid and B: $100 \% \mathrm{MeOH}$. The gradient (from $30 \%$ to $80 \%$ of B in $3 \mathrm{~min}$ ) was eluted at a flow rate of $0.31 \mathrm{~mL} / \mathrm{min}$. Source voltage: $2000 \mathrm{~V}$; sample cone: $30 \mathrm{~V}$; desolvation: $350^{\circ} \mathrm{C}$; source: $120^{\circ} \mathrm{C}$; Gas Flow Cone: $10 \mathrm{~L} / \mathrm{h}$; desolvation gas: $400 \mathrm{~L} / \mathrm{h}$; using the full scan mode and a m/z range of $100-1000$.

\subsection{Dead Sea Water (DSW) Extract}

The major constituents of DSW (Osmoter ${ }^{\mathrm{TM}}$ by AHAVA) are the following ions: $\mathrm{Mg}^{2+}(92,500 \mathrm{mg} / \mathrm{L}), \mathrm{Ca}^{2+}(38,000 \mathrm{mg} / \mathrm{L}), \mathrm{K}^{+}(1400 \mathrm{mg} / \mathrm{L}), \mathrm{Na}^{+}(2000 \mathrm{mg} / \mathrm{L}), \mathrm{Sr}^{2+}$ $(800 \mathrm{mg} / \mathrm{L}), \mathrm{Cl}-(345,000 \mathrm{mg} / \mathrm{L})$ and $\mathrm{Br}-(11,500 \mathrm{mg} / \mathrm{L})$. 


\subsection{Human Skin Organ Culture for Biological Tests}

EX vivo human skin organ culture (HSOC) [19] was used as a representative skin laboratory model for testing $C$. procera extract safety threshold and its protection against inflammation and irritation.

Human skin cultures were obtained from healthy females (age 23 - 45) undergoing abdominal plastic surgery. The study was initiated at the day of surgery. Fixed size of skin pieces $\left(0.64 \mathrm{~cm}^{2}\right)$ were cut from the skin tissue, using a designated press apparatus. The skin pieces were placed in culture medium (DMEM supplemented with $100 \mathrm{U} / \mathrm{ml}$ penicillin and $100 \mu \mathrm{g} / \mathrm{ml}$ streptomycin), dermal side down in the medium and epidermis up. The pieces were incubated overnight at $37^{\circ} \mathrm{C}$ with $5 \% \mathrm{CO}_{2}$ for recovery for $24 \mathrm{hr}$.

\subsection{Treatment with $C$. procera Callus Extracts for Dose Response Analysis}

After recovery, $C$. procera callus extract was applied on the skin pieces topically at five different concentrations by a serial dilution of 1:10, 1:50, 1:100, 1:500, 1:5000. Topical application of $3 \mu \mathrm{l}$ was mounted on the skin. The dilution of the C. procera callus extract was carried out in its original formulation i.e., Glycerol:DDW (80\%:20\%) mixture containing C. procera at a concentration of $40 \mathrm{~g} / \mathrm{L}$, as in detailed in the formulation section.

The Control group represents Naïve skin. Vehicle treated group (glycerol:water) served as negative control. 10\% SDS and UVB $\left(400 \mathrm{~mJ} / \mathrm{cm}^{2}\right)$ served as positive control for cell viability and apoptois respectively.

The skin pieces were incubated for 24,48 and $72 \mathrm{hr}$ at $37^{\circ} \mathrm{C}$ with $5 \% \mathrm{CO}_{2}$.

After 24- and 48-hr, the second and third time point incubation groups were applied similarly. At the end of all incubations, the epidermis was peeled and its viability and apoptosis were evaluated.

\subsection{Inflammation Induction by Lipopolysaccharides (LPS) and Treatment with $C$. procera Extracts}

Fixed size of skin pieces $\left(0.64 \mathrm{~cm}^{2}\right)$ was cut from the skin tissue, using a designated press apparatus. The skin pieces were laid in 6-well culture plates containing skin culture medium (DMEM supplemented with $100 \mathrm{U} / \mathrm{ml}$ penicillin and $100 \mu \mathrm{g} / \mathrm{ml}$ streptomycin), dermal side down in the medium and epidermis up. The pieces were incubated overnight at $37^{\circ} \mathrm{C}$ with $5 \% \mathrm{CO}_{2}$ for recovery for $24 \mathrm{hr}$. To induce inflammation characteristics, fresh culture medium was supplemented with LPS $(10 \mu \mathrm{g} / \mathrm{ml})$, which was added to the skin pieces after recovery. Culture medium without supplements was used as negative, unstimulated control. In addition, glycerol:DDW (80:20) mixture was used as vehicle control group.

The dilutions of the $C$. procera extract were carried out in their original formulation, i.e., Glycerol: DDW (80\%:20\%) mixture. Naïve and LPS-stimulated cultures were treated without or with three non-toxic concentrations of the ex- 
tracts by applying them on the epidermis topically $(3 \mu \mathrm{L}) 15$ min prior to LPS stimulation. These concentrations were made by a serial dilution of 1:50, 1:100, 1:200. The positive control contained LPS, without addition of other agents.

The pieces were incubated for $48 \mathrm{hr}$ at $37^{\circ} \mathrm{C}$ with $5 \% \mathrm{CO}_{2}$. At the end of incubation the epidermis was peeled and its viability was evaluated by MTT assay. Concomitantly, spent medium from treated skin cultures was collected under standardized conditions $(\sim 1000 \mu \mathrm{l})$ and centrifuged at $1500 \mathrm{~g}$ for $5 \mathrm{~min}$ to remove particulates and cells. Clear supernatants were frozen at $-70^{\circ} \mathrm{C}$ for cytokines (IL-1 $\beta, \mathrm{TNF} \alpha$ ) quantification.

\subsection{Irritation Induction by Sodium Dodecyl Sulfate (SDS) and Treatment with $C$. procera Extracts}

Fixed size of skin pieces $\left(0.64 \mathrm{~cm}^{2}\right)$ was cut from the skin tissue, using a designated press apparatus. The skin pieces were laid in 6-well culture plates containing skin culture medium (DMEM supplemented with $100 \mathrm{U} / \mathrm{ml}$ penicillin and $100 \mu \mathrm{g} / \mathrm{ml}$ streptomycin), dermal side down in the medium and epidermis up. The pieces were incubated overnight at $37^{\circ} \mathrm{C}$ with $5 \% \mathrm{CO}_{2}$ for recovery for $24 \mathrm{hr}$. To induce irritation, $10 \% \mathrm{w} / \mathrm{w}$ of Sodium Dodecyl Sulfate (SDS) was applied topically $(3 \mu \mathrm{l}) .15 \mathrm{~min}$ later, the cultures were treated without or with three concentrations of non-toxic concentrations of the extracts by applying them on the epidermis topically $(3 \mu \mathrm{L}) 15$ min prior to LPS stimulation. These concentrations were made by a serial dilution of 1:200, 1:100, 1:50. The pieces were incubated for $48 \mathrm{hr}$ at $37^{\circ} \mathrm{C}$ with $5 \% \mathrm{CO}_{2}$. At the end of incubation the epidermis was peeled and its viability was evaluated by MTT assay. Concomitantly, spent medium from treated skin cultures was collected under standardized conditions ( $1000 \mathrm{l}$ ) and centrifuged at $1500 \mathrm{~g}$ for $5 \mathrm{~min}$ to remove particulates and cells. Clear supernatants were frozen at $-70^{\circ} \mathrm{C}$ for cytokines (IL- $1 \beta, \operatorname{TNF} \alpha$ ) quantification and Prostaglandin $\mathrm{E}_{2}\left(\mathrm{PGE}_{2}\right)$ determination.

\subsection{MTT Cell Viability Assay}

Viability was evaluated by mitochondrial reduction using, 5-dimethylthiazol-2-yl]-2,5-diphenyl tetrazolium bromide (MTT) assay. Epidermis units were treated with $1 \mathrm{mg} / \mathrm{mL}$ MTT solution for 3 hours at $37^{\circ} \mathrm{C}$. The solution was then removed and replaced with isopropanol, with further 2 hours incubation at room temperature. Two aliquots of every sample were transferred to a 96-well plate for the reading. The absorbance was read at the wavelength of $540 / 570 \mathrm{~nm}$ with a colorimeter equipped with a microplate reader Fluoroskan Ascent spectrofluorimeter (Thermo Scientific).

\subsection{Cell Apoptosis by Caspase 3 Activity}

Apoptosis determination by caspase 3 assay was carried by caspase- 3 activity measurement as previously described [10]. The collected epidermis pieces were 
moved into a 96-well plate, each well filled by $125 \mu \mathrm{L}$ of aspase- 3 specific substrate solution $(10 \mu \mathrm{M})$ Ac-DEVD-AMC; Merck, Darmstadt, Germany) with $0.02 \%$ Triton X-100 (J. T. Baker, Phillipsburg, NJ, USA) and $10 \mathrm{~mm}$ DTT (TCI, Tokyo, Japan), at $37^{\circ} \mathrm{C}$ in a 96 -well plate. The enzyme's fluorescent product emission level $(355 / 460 \mathrm{~nm})$ was measured at 2-min intervals using fluorescence plate reader Fluostar-BMG spectrofluorimeter at $37^{\circ} \mathrm{C}$ for $40 \mathrm{~min}$. Apoptosis level was later deduced from the slope value of enzymatic activity in the linear range.

\subsection{Cytokines and Prostaglandin Quantification}

Cytokines quantification of skin culture supernatants were analyzed with specific ELISA kits for TNF $\alpha$, IL- $1 \alpha$ and IL- $1 \beta$ (Biolegend) and for Prostaglandin $\mathrm{E}_{2}$ ( $\mathrm{PGE}_{2}$, Enzo Life Sciences) [8]. Briefly, wells in 96-well plates were coated with capture antibody. Following blocking, spent media as well as standard cytokine solutions were incubated in the coated wells. Wells were washed and biotinylated detection antibody solution was incubated in the wells. Following additional washes, extravidin-peroxidase conjugate solution was incubated in the wells. The HRP fluorogenic substrate QuantaBlue (Thermo Scientific, Rockford, IL, USA) was added to the wells and emission was measured using a Fluoroskan Ascent spectrofluorimeter (Thermo Scientific) fluorescence plate reader (320/405 $\mathrm{nm}$ ) every $2 \mathrm{~min}$ for $40 \mathrm{~min}$. The level of peroxidase activity was deduced from the slope value of enzymatic activity in the linear range. The level of the cytokines in the spent media was deduced from a standard curve based on the activity levels of the standard cytokine solutions.

\subsection{Reconstructed Skin Model}

MatTek Full Thickness Tissues were placed into a 6-well plate containing $2.5 \mathrm{ml}$ of assay medium and incubated overnight at $37^{\circ} \mathrm{C} \pm 2^{\circ} \mathrm{C}$ and $5 \% \pm 1 \% \mathrm{CO}_{2}$ [8].

Then, the assay medium was replaced with $5 \mathrm{ml}$ of fresh medium $\left(37^{\circ} \mathrm{C} \pm 2^{\circ} \mathrm{C}\right)$ and the tissues were treated topically with $0.4 \mathrm{~g} / \mathrm{L}$ C. procera extract alone, $5 \mathrm{~g} / \mathrm{L}$ DSW alone or with the combination of $0.4 \mathrm{~g} / \mathrm{L} C$. procera extract and $5 \mathrm{~g} / \mathrm{L}$ DSW for 48 hours. At the end of the incubation period the surface of the tissues were rinsed with PBS to remove the test materials after which the tissues were homogenized (for RNA/proteins isolations) and the tissue culture media was collected (for ELISA methods).

\subsection{RNA Isolation}

Total RNA was extracted from $48 \mathrm{~h}$ treated MatTek EFT-400 full thickness skin tissues by RNAqueous kit (Ambion) followed by mRNA Amplification protocol (Ambion MessageAmp aRNA kit). Total RNA was reverse-transcribed to cDNA using a T7 oligo (dT) primer. Second-strand cDNA was synthesized and in vitro transcribed to aRNA. The RNA concentration was determined by Ribogreen assay reagent (Molecular Probes) using Thermo Labsystems Fluorskan Ascent FL 
fluorometer and the RNA quality was evaluated via gel electrophoresis.

\subsection{Microarray Experiment}

aRNA probes from untreated, $C$. procera extract alone, DSW alone or with the combination of $C$. procera extract and DSW samples were labeled with Cy3 or Cy5, respectively, using PerkinElmer ASAP RNA Labeling Kit and Purified by Millipore Microcon YM-30 filter column and TE buffer. After purification, the fluorescent aRNA probes prepared above were hybridized to DNA Microarray SureHyb Chip (Agilent Technologies) for $17 \mathrm{~h}$ at $65^{\circ} \mathrm{C}$. The microarrays signal was scanned by Axon GenePix 4100A Scanner with the scanning resolution set to $5 \mu \mathrm{m}$ and analyzed with GenePix Pro software. During the initial scan the PMT gains for the scanner were adjusted such that the cy5/cy3 image count ratios were between 0.95 and 1.05. Fluorescence intensities for the microarrays were subjected to global normalization by quantile method and were express as adjusted $\log$ FCs [8].

\subsection{Gene Set Enrichment Analysis (GSEA)}

The GSEA software was downloaded from the Broad Institute (http://software.broadinstitute.org/gsea/index.jsp). The adjusted logFCs were used for ranking the whole transcriptome and GSEA (cut-off independent) was carried out as described previously (Subramanian et al., PNAS 2005) using the MSigDB v6.1 database category $\mathrm{H}$. Enrichment of gene sets was considered statistically significant if the false discovery rate (FDR) was $<0.05$.

\subsection{Immunoblotting}

The Full Thickness Tissues were lysed in CelLytic MT Cell Lysis Reagent that were supplemented with protease inhibitor cocktail and proteins were resolved on $12 \%$ reduced SDS-PAGE and subjected to immunoblotting using the following specific Abs: anti-phosphofructokinase (PFK, F-7 clone, Santa Cruz Biotechnology), anti-Hypoxia Inducible Factor 1 (HIF1, H1alpha67 clone, EMD Millipore) and anti-GAPDH (Sigma Aldrich), which was used to normalize the data followed by secondary fluorophore antibody (Abcam). Detection was performed by BioRad Molecular Imager FX and Images analyzed using ImageJ image analysis software.

\subsection{Fibronectin ELISA}

Fibronectin quantification of skin culture supernatants were analyzed with specific ELISA kit (Takara Bio USA). Briefly, a series of fibronectin standards was prepared ranging from $0 \mathrm{ng} / \mathrm{ml}$ to $800 \mathrm{ng} / \mathrm{ml}$. Next, $100 \mu \mathrm{l}$ of either sample (collected Full Thickness Tissues culture media) or standard was then added to appropriate wells followed by the addition of $100 \mu \mathrm{l}$ a peroxidase-labeled anti fibronectin antibody. Next, $100 \mu \mathrm{l}$ of peroxidase substrate solution (hydrogen peroxide + tetramethylbenzidine as a chromagen) was added to each well followed 
by the addition of $100 \mu \mathrm{l}$ of stop solution ( $1 \mathrm{~N}$ sulfuric acid). The plate was read using a microplate reader at $450 \mathrm{~nm}$.

\subsection{Statistical Analysis}

Averaged values are presented as the mean \pm SEM. When comparing two groups, statistical significance was determined using a one way ANOVA or a two-tailed Student $t$ test. $p$ value $<0.05$ was considered statistically significant.

\section{Results}

\subsection{Phytochemical Analysis of Calotropis procera Callus Extract}

Callus extract of C. procera was generated by hot water extraction and detailed chemical analysis as performed with a combination of HPLC-DAD and UPLC-MS. Previously identified toxic compounds from Calotropis plant species, along with their molecular mass are Calotropin (523.63 Da), Calactin (523.63 Da), Calotoxin (548.63 Da), Uscharidin (530.61 Da), Calotropagenin (404.50 $\mathrm{Da})$, Uscharin (587.67 Da), Gigantin (154.12 Da), 3'O acetyl calotropin (synonyme of asclepin, 574,67 Da), Calotropin 3' glucoside (694.77 Da), Calotropone (468.59 Da), Calotroposide A and B (1189.44 Da and 1205.44 Da), Frugoside (536.66 Da), Proceroside (548.63 Da), Uzarigenin (374.52 Da), Voruscharin (589.74 Da) and 2'Oxovoruscharin (603.73 Da) [2].

The sustainable generation of $C$. procera biomass using plant biotechnology had a strong impact on the phytochemical profile of the water-based extract, as none of the known toxic compounds was present in detectable amount in the extract. We could only detect one compound (data not shown) with a molecular mass of $520 \mathrm{Da}$ which could correspond to an unknown cardiac glycoside previously described in a close relative plant species, Calotropis gigantea [21], suggesting a potential lower toxicity of such callus extract than the plant itself.

\subsection{Dose Response for Safety Threshold}

The aim of this experiment was to determine the concentration and time range, where each $C$. procera extract tolerated by the skin without causing a decrease in epidermis vitality.

Figure 1 shows the dose-response and time course analyses in terms of viability and apoptosis. Figure 1(a) shows the impact of the different test items on cell viability by MTT. Except of SDS ( $10 \% \mathrm{vol} / \mathrm{vol})$, which served as a positive control and significantly reduced viability in all time points by $41 \%-58 \%$, no significant effect was detected by all $C$. procera extracts and their vehicle.

Figure 1(b) shows the impact of the different test items on Caspase- 3 activation, which is indicative to apoptosis. Other than the positive control group (UVB), which significantly enhanced caspase 3 activity in all time points by 20 100 fold, all $C$. procera extracts and their vehicle detected no significant effect.

These results indicate that $C$. procera extracts at concentration between $4 \mathrm{~g} / \mathrm{L}$ $8 \mathrm{mg} / \mathrm{L}$ have no effect on cell viability and apoptosis. 


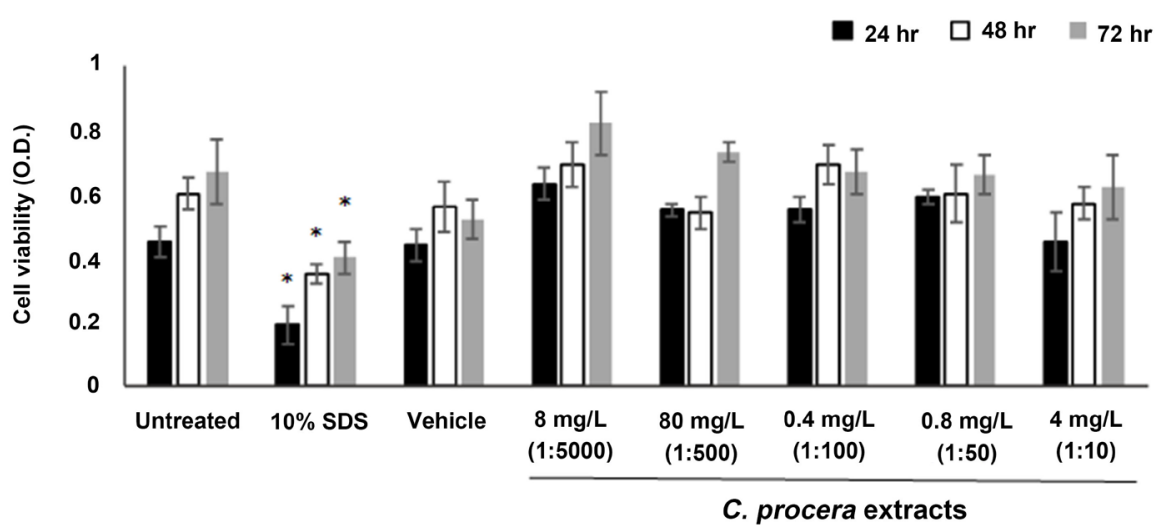

(a)

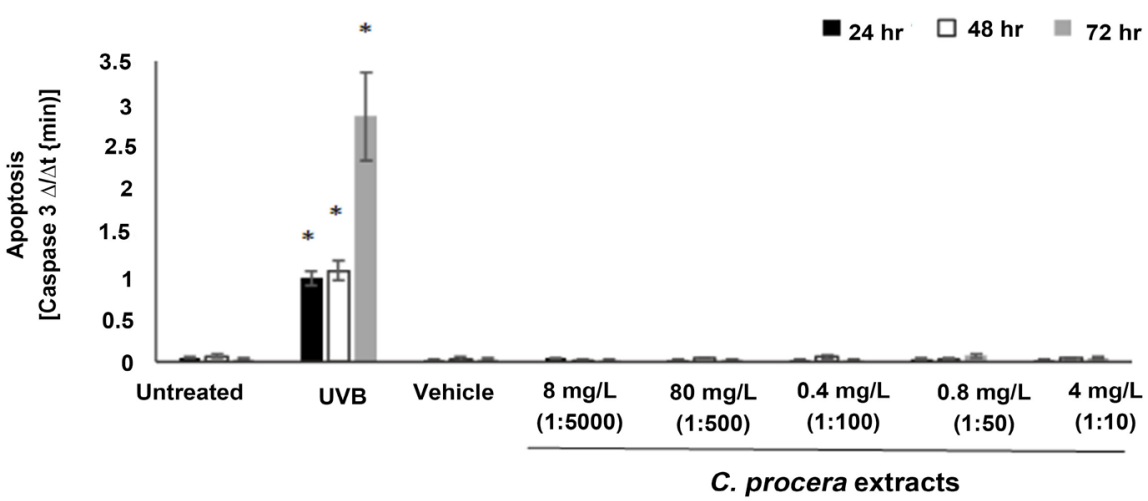

(b)

Figure 1. Dose response analysis of $C$. procera extracts for skin safety threshold. The HSOC pieces were incubated without or with $C$. procera different extract concentrations for 24-, 48- and 72-hr. Then, epidermis viability was measured by MTT assay (a). Epidermal apoptosis was evaluated by caspase-3 activity kinetically (b). Values are expressed as means $\pm \mathrm{SEM}$; ${ }^{*} \mathrm{p}<0.05$ for differences from Control. SDS $=$ positive control for viability. UVB $=$ positive control for apoptosis.

\subsection{Biological Activity of $C$. procera Extract against Skin Inflammation and Irritation}

Based on the dose response tests results, three different $C$. procera extracts concentrations, which did not affect cells viability or apoptosis were selected: 0.2 $\mathrm{g} / \mathrm{L}, 0.4 \mathrm{~g} / \mathrm{l}, 0.8 \mathrm{~g} / \mathrm{L}$. These concentrations were tested for a protective effect against inflammation and irritation.

Inflammation was performed by LPS $(10 \mu \mathrm{g} / \mathrm{ml})$ addition to medium. Although LPS did no affect cell viability (Figure $2(a)$ ), the results show a significant 3 fold and 4 fold induction of the inflammatory cytokines IL- $1 \beta$ and TNF $\alpha$ respectively than the control (Figure 2(b), Figure 2(c)). Treatment with C. procera extracts at concentrations of $0.4 \mathrm{~g} / \mathrm{L}, 0.8 \mathrm{~g} / \mathrm{L}$ significantly attenuated IL- $\beta$ induction by $42 \%$ and $57 \%$ respectively (Figure $2(\mathrm{~b})$ ). These two concentrations also significantly attenuated TNF $\alpha$ secretion by $24 \%$ and $35 \%$ (Figure $2(\mathrm{c})$ ). Unlike $C$. procera extracts attenuation effect, treatment with their vehicle did not attenuate cytokines secretion induced by LPS. 


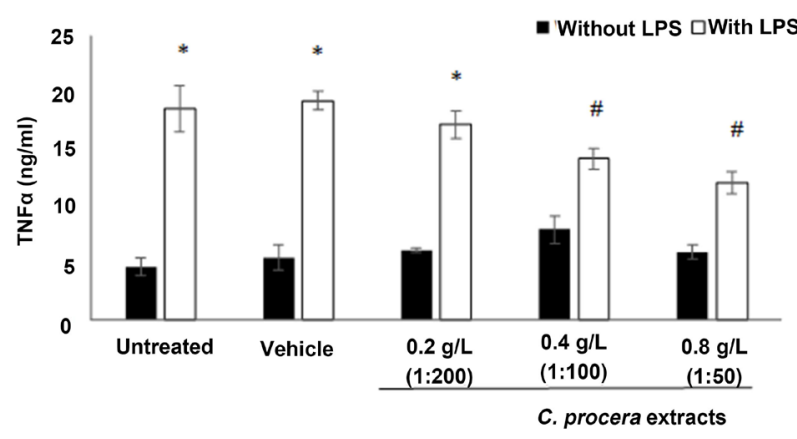

(a)

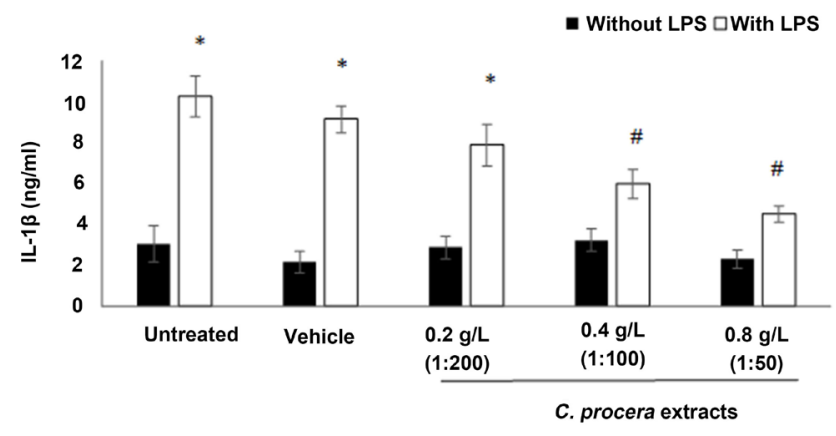

(b)

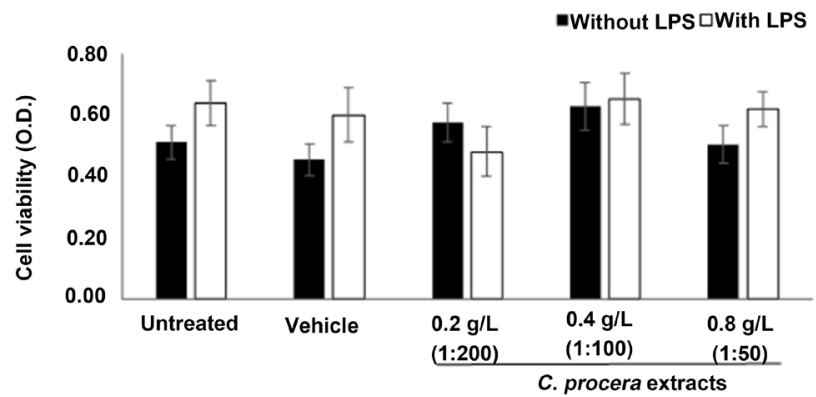

(c)

Figure 2. The impact of C. procera extract on LPS-induced inflammation. The HSOC pieces were incubated with LPS (10 $\mu \mathrm{g} / \mathrm{ml})$ to induce inflammation. Concomitantly, the explants were treated without or with the indicated concentrations for $48 \mathrm{hr}$. Then, TNF $\alpha$ (a) and IL-1 $\beta$ (b) levels in the spent medium were evaluated by ELISA. In addition, epidermis viability was measured by MTT assay (c). Values are expressed as means \pm SEM. ${ }^{*} \mathrm{p}<0.05$ for differences from the control.; \# $<0.05$ for differences from the LPS-treated control.

Irritation was induced by SDS $(10 \% \mathrm{w} / \mathrm{w})$ topical application. As shown in Figure 3(a), SDS application led to a significant decrease in epidermal viability by $47 \%$ in untreated skin. Treatment with all $C$. procera extracts significantly abolished this viability reduction. SDS application also enhanced significantly the irritation-induced cytokines, IL- $\alpha$ and TNF $\alpha$, by 4 fold and 2.5 fold than the untreated (control) respectively (Figure 3(b), Figure 3(c)). Treatment with $C$. procera extracts at concentrations of $0.4 \mathrm{~g} / \mathrm{L}, 0.8 \mathrm{~g} / \mathrm{L}$ significantly attenuated the induction of IL- $1 \alpha$ by 2 fold (Figure 3(b)). Additionally to inflammatory cytokine secretion, the lipid, prostaglandin $2\left(\mathrm{PGE}_{2}\right)$, secretion was significantly induced by 2.8 fold following topical application of SDS than control (Figure 3(d)). Treatment with $C$. procera extracts at concentrations of $0.4 \mathrm{~g} / \mathrm{L}$ and 0.8 g/L significantly attenuated $\mathrm{PGE}_{2}$ induction by $40 \%$.

Unlike the effect of $C$. procera extract, treatment with their vehicle neither attenuated the induction of cytokines and $\mathrm{PGE}_{2}$ nor abolished the reduction in epidermis viability.

\subsection{Biological Activity of $C$, procera Extract in Combination with DSW Extract on Skin}

In the second part of the study $C$. procera extract was combined with another Dead Sea regional active ingredient, Dead Sea water (DSW) to test their effect on 


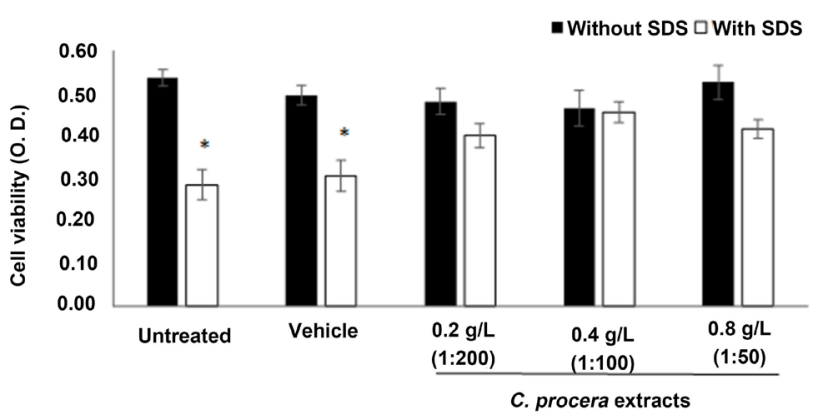

(a)

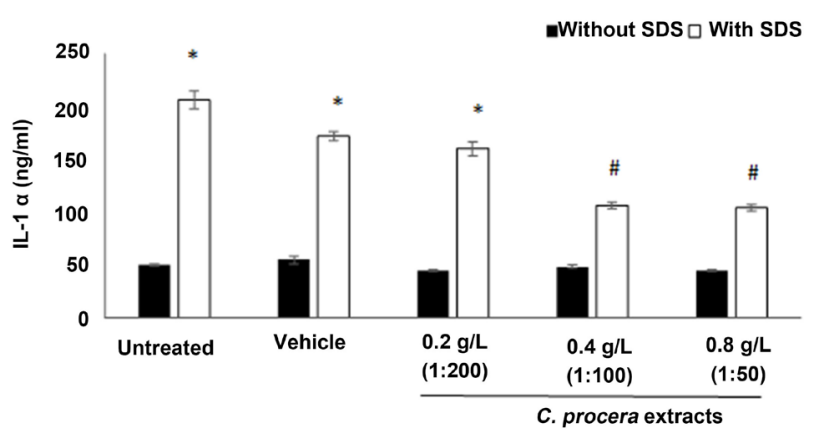

(c)

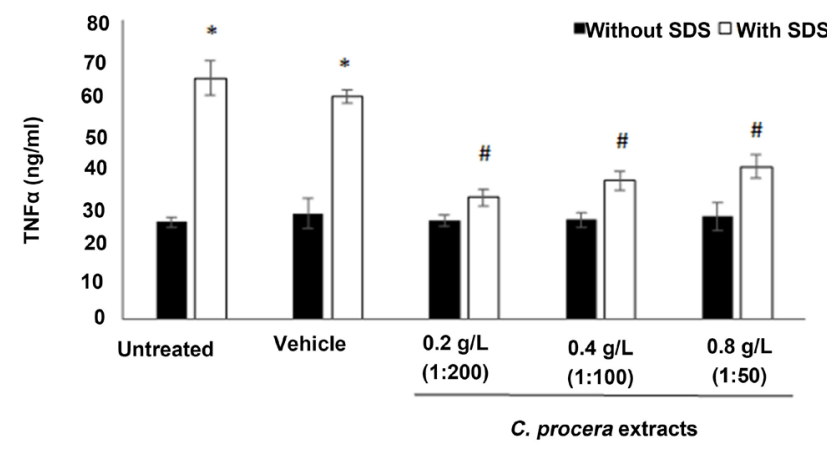

(b)

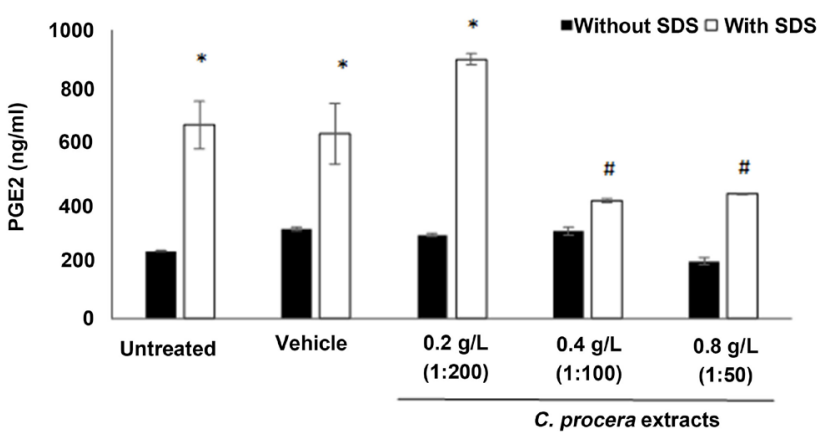

(d)

Figure 3. The impact of $C$. procera extract on SDS-induced irritation. The HSOC pieces were incubated with $10 \%$ SDS (vol/vol) to induce irritation. Concomitantly, the explants were treated without or with the indicated concentrations for $48 \mathrm{hr}$. Then, epidermis viability was measured by MTT assay (a). In addition, TNFa (b), IL-1 $\alpha$ (c) and Prostagland in $\mathrm{E}_{2}$ (PGE P $_{2}$ (d) levels in the spent medium were evaluated by ELISA. Values are expressed as means \pm SEM and normalized to cell viability. ${ }^{*} \mathrm{p}<0.05$ for differences from the Vehicle control; \# $\mathrm{p}<0.05$ for differences from the SDS-treated control.

skin gene expression. The effects of these extracts on various biological functional gene sets were analyzed by the GSEA approach. To this end, $0.4 \mathrm{~g} / \mathrm{L} C$. procera extract and $5 \mathrm{~g} / \mathrm{L}$ DSW extract alone or in combination were topically applied on reconstructed full thickness skin tissues. Comparison of the gene profiles of the treatment groups to whole transcriptome GSEA revealed that biological processes pertaining to hypoxia (Figure 4(a)), glycolysis (Figure 4(b)) and epithelial mesenchymal transition (EMT, Figure 4(c)) were enriched. The GSEA analyses further confirmed that those three biological pathways were found to have an opposite direction in the combined treatment group versus treatment with each ingredient as stand-alone. In the treatment group with a combination of C. procera and DSW all three biological pathways were significantly down-regulated (hypoxia FDR $=0$, glycolysis FDR $=0.036$ and EMT FDR $=0$ ) while in the treatment groups with $C$. procera or DSW separately, the three biological pathways were up-regulated (hypoxia: $\mathrm{FDR}=0.014$ for DSW and FDR $=0$ for $C$. procera extract, glycolysis: FDR $=0.021$ for DSW and FDR $=0.11$ for C. procera extract and EMT FDR $=0.022$ for DSW and FDR $=0.077$ for $C$. procera extract).

The above-mentioned enriched biological pathways flowed by C. procera extract + DSW were further tested in the protein level by evaluating representative 
DSW

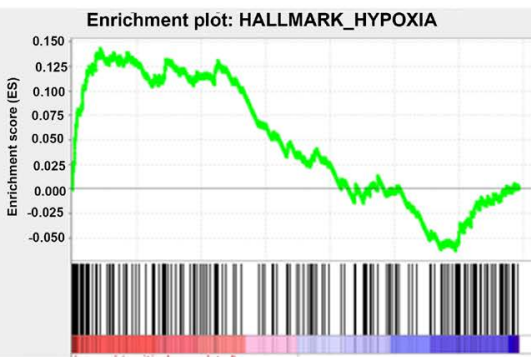

DSW

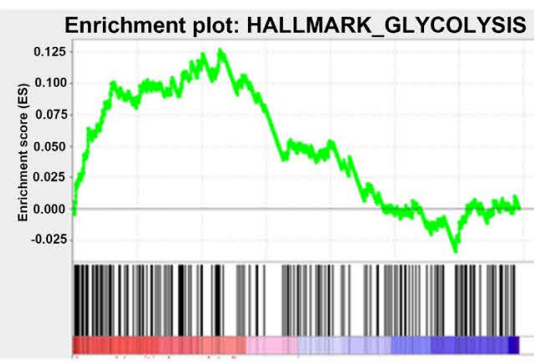

DSW
C.Procera

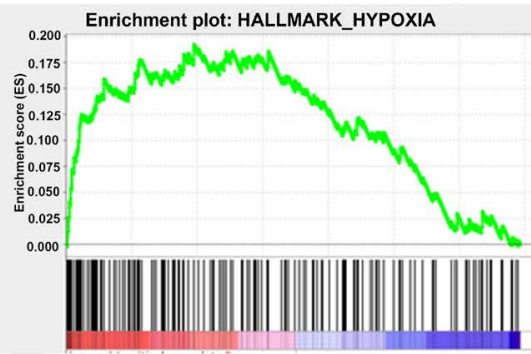

(a)

C. Procera

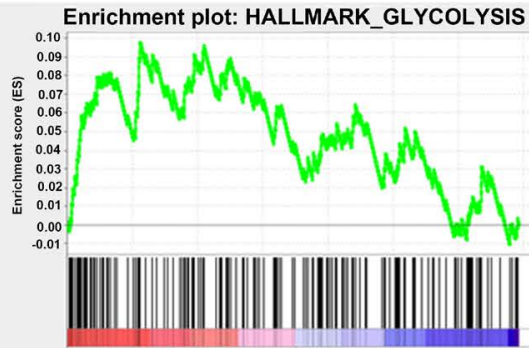

(b)
C.Procera + DSW

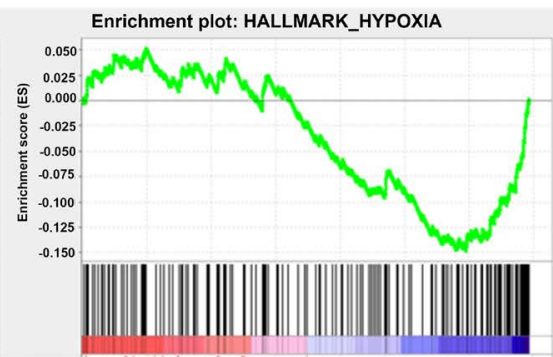

C. Procera + DSW

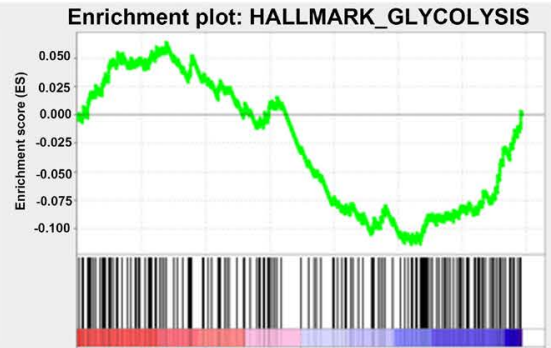

C.Procera

C.Procera + DSW

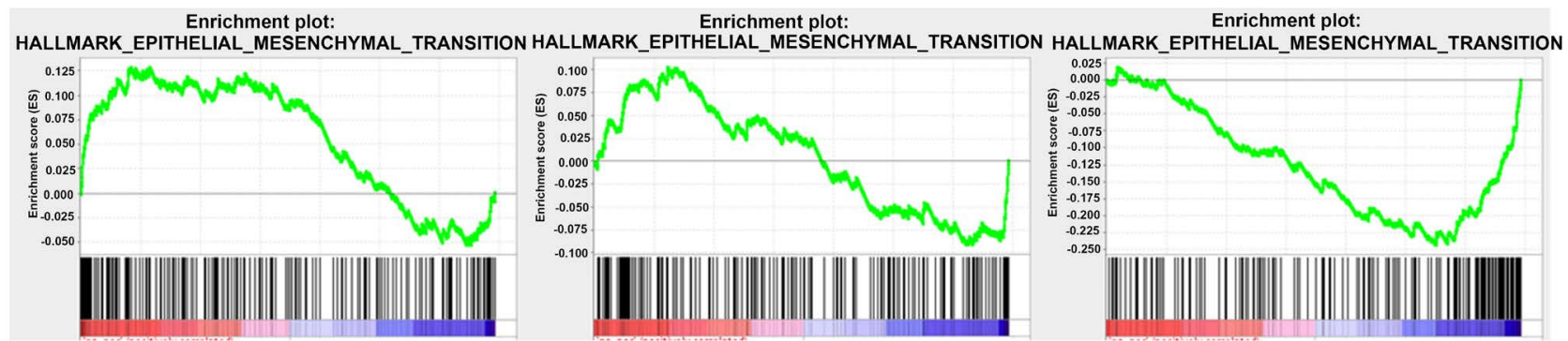

(c)

Figure 4. Gene Set Enrichment Analysis (GSEA) in reconstructed skin upon treatment with $C$. procera extract and DSW. MatTek full thickness reconstructed skin were treated with $C$. procera extract alone (left panels), DSW alone (middle panels) or $C$. procera extract plus DSW (right panels) and subjected to GSEA for (a) Hypoxia; (b) Glycolysis and (c) Epithelial Mesenchymal Transition cellular pathways $(\mathrm{FDR}<0.05)$.

protein/enzymatic biomarkers for each pathway. To investigate the role of hypoxia pathway in human skin under the combined treatment of $C$. procera extract + DSW, the expression of hypoxia-inducible factor 1 (HIF1) was measured. HIF1 is a ubiquitously expressed heterodimeric transcription factor, which is known to be associated with mammalian cells ability to adapt hypoxia conditions [22] and involved also in skin biological processes, including wound healing and infections. Using immunoblotting approach, a significant increase expression of HIF1 by $15 \%$ was observed only after treatment with a combination of $C$. procera extract + DSW, unlike the untreated skin or skin treated with each 
of the tested materials alone (Figure 5(a)). Glycolysis pathway was evaluated by the same experimental methodology for the marker Phosphfructokinase-1 (PFK1), a key enzyme involves the hydrolysis of ATP, a critical step in determining glycolytic flux and has been correlated with changes in cellular metabolism and physiology [23]. The results show a significant enhancement in PFK1 expression by $20 \%$ when skin was topically applied with combination of C. procera extract + DSW extract relatively to untreated skin or when treated separately with each of the tested ingredients alone (Figure 5(b)). The ability of $C$. procera extract and DS water extract to affect EMT physiologic processes was assessed by evaluating the amount of fibronectin secreted to culture medium. Fibronectin is a key master of the extracellular matrix (ECM), forming essential connections between cell surface integrin receptors and structural components of the ECM, enhancing skin cells differentiation, assisting in regeneration and healing [24]. Figure 5(c) indicates that both topical treatment with C. procera extract alone and the mixture of $C$. procera extract and DSW extract were observed to significantly increase fibronectin production by $22 \%$ and $20 \%$, respectively.

\section{Discussion}

The fear from skin appearance of an old person is the engine of cosmetic industry and therefore, anti-aging products are the gold core of the huge worldwide cosmetic business. The anti-aging cosmetology main perception focus for many years had been on diminishing the typical skin aging symptoms or hiding them using a long list of skincare preparations, including products targeting skin deep wrinkles, fine-lines, age spots, discoloration, loss of skin young appearance related to reduction of moisture, elasticity and glow. Recently, a new anti-aging strategy has been evolved, proposing to fight against the biological causes beyond the appearance of symptoms, i.e. trying to reduce the damage of natural processes involved in its cellular level.

Skin aging reflects the accumulation of damaged molecules following skin exposure to a wide range of stressors [25] [26]. This unavoidable accumulation leads to an activation of "pro-aging" biochemical and metabolic pathways that could eventually result with the appearance of skin aging symptoms or activate skin pathologies [16] [17] [18]. Inflammatory processes are recognized as key mediators of the different pathways leading to skin aging. These process are characterized by enhancement inflammatory cytokine secretion (e.g. IL-1 $\alpha$, IL-6, IL-8, TNF $\alpha$ ), matrix metalloproteinase (MMPs) activation and resulting in accumulation of reactive oxygen species (ROS) [10] [26]. Therefore, lots of research efforts target attenuation of skin inflammation while skin models, such as reconstructed in-vitro skin and human ex-vivo donated skin are used as important research tools [8] [9] [10] [19].

The new anti-aging strategy, coping not only with skin symptoms, but rather, aiming to affect the biological mechanisms beyond symptoms appearance, has 


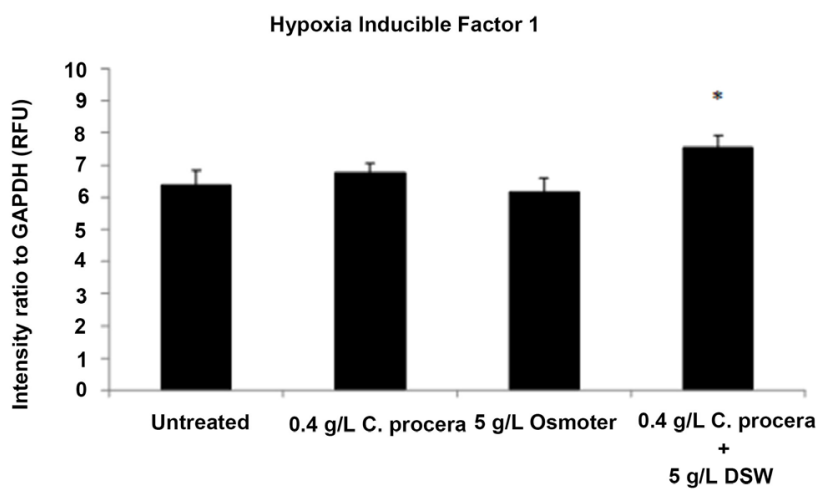

(a)

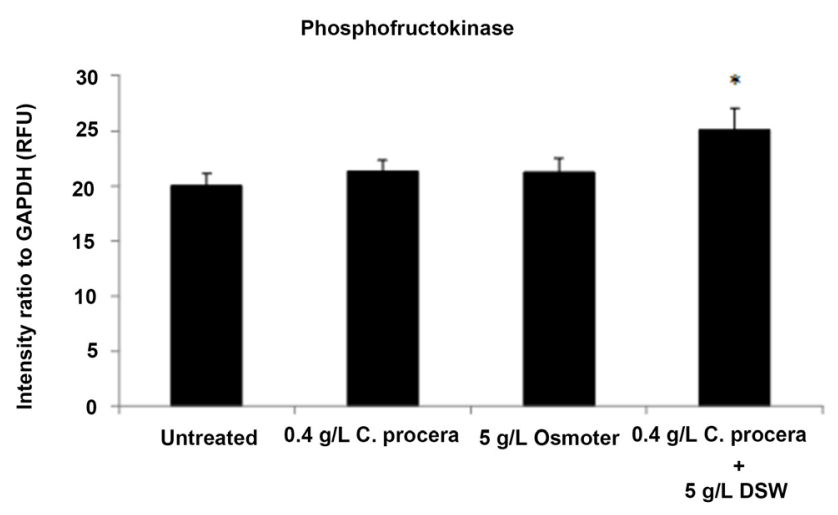

(b)

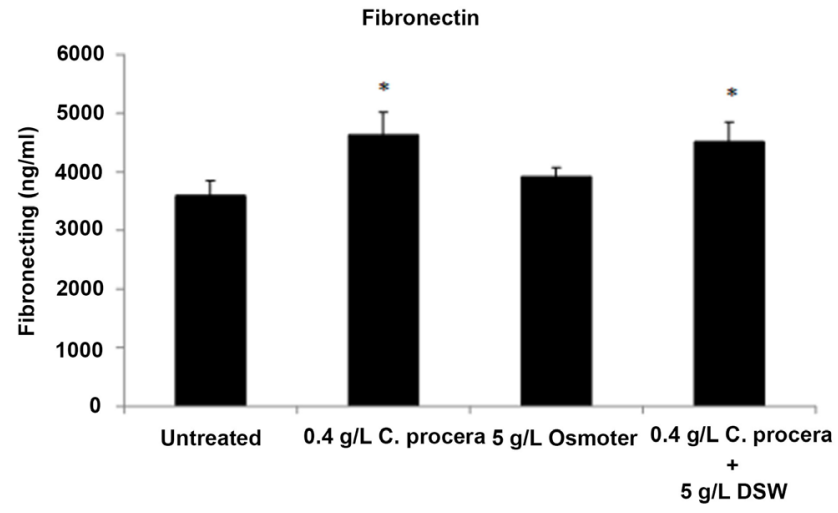

(c)

Figure 5. DSW and C. procera extract combined treatment induced skin related biological activities. MatTek full thickness reconstructed skin was treated without or with $C$. procera extract, DSW extract and their combination for $48 \mathrm{~h}$. Then, tissues were homogenized and subjected to immunoblotting for testing (a) Hypoxia Inducible Factor 1 and (b) Phosphofructokinase expression levels. Simultaneously, the tissue culture media was collected and tested for Fibronectin accumulation (c) by ELISA. Values are expressed as mean RFU \pm SEM normalized to GAPDH expression $((\mathrm{a})+(\mathrm{b}))$ or as $\mathrm{ng} / \mathrm{ml}(\mathrm{c}) .{ }^{*} \mathrm{p}<0.05$ for differences from the untreated control.

been evolved due to a better understanding of biological pathways in different levels as well as selection of more potent actives to affect the relevant biological mechanism. Many skin active ingredients are derived from botanical sources including plant extracts and oils. Some of the plants that are extracted known to be toxic, yet when formulated in moderate dose, i.e. small concentrations, are not poisoning and exhibit positive therapeutic skin effects [27]. This phenomenon is in line with the theory of "hormesis", which takes "toxic known substance" to be utilized as beneficial, when used in smaller concentrations [28]. Hence, more sophisticated treatment strategy can be designed by exploiting a diversity of substances considered as toxic. Furthermore, a combination of several substances might lead to additional positive outcomes, which were not observed on each ingredient as stand-alone.

The use of $C$. procera plant extract is an excellent example for taking a toxic plant and making it beneficial by treatment with small doses. Calotropis procera, grows in Dead Sea natural environment as a protected plant. Choosing Callus technology for plant extraction, in addition to attenuate the expression of toxic 
compounds, enable a controlled preparation of big amounts of extract in well-ordered quality without threatening the prevalence of a natural specie of its local flora. Plant extraction via callus technology is in line with modern trends and sustainable approach.

The new $C$. procera callus extract was then tested for biological activity on $e x$ vivo human skin models, which might serve as a predictive tool. a. Different extract dilutions at concentrations from $4 \mathrm{~g} / \mathrm{L}$ to $8 \mathrm{mg} / \mathrm{ml}$ were tested for safety. All of them did not show decrease in vitality parameters (viability and apoptosis).Three extract concentrations, at the safe range (Figure 1) were chosen for further activity testing in terms of protection against inflammation and irritation, common skin manifestations also relevant to cosmetic treatment. Acceptable models for inflammation and irritation induction in laboratory are by the addition of LPS and SDS respectively [29] [30]. Still, clinical tests should be performed on human volunteers in order to support safety and activity claims.

The LPS-induced skin inflammation is an experimental system to observe an inflammatory response, imitating bacteria invade, known to induce the secretion of the cytokines TNF $\alpha$ and IL-1 $\beta$ [31] [32]. Indeed, the obtained results following adding LPS using human skin cultures model demonstrated a significant increase in TNF $\alpha$ and IL-1 secretion (Figure 2). Treatment with $C$. procera extract significantly attenuated these inflammatory markers using concentrations of 0.8 and $4 \mathrm{~g} / \mathrm{L}$, while vehicle control did not demonstrate any protective effect. Thus, we conclude $C$. procera extract possess anti-inflammatory properties.

The SDS-induced skin irritation model is an experimental system allows monitoring the ability of the tested item to attenuate the deleterious impact of the SDS strong detergent of the irritant on skin surface. The hallmark of this system is the reduction of epidermis viability and the concomitant induction of specific inflammatory markers, particularly IL- $1 \alpha, \mathrm{TNF} \alpha$ and $\mathrm{PGE}_{2}$ [33] [34]. As expected, the viability decreased and the levels of three measured inflammation markers were significantly increased, following SDS treatment (Figure 3). In addition, the vehicle control was not differing from the untreated control. Treatment with $C$. procera extract significantly abolished the decrease in epidermal viability and attenuated the elevation in all three tested biomarkers. These results indicate that $C$. procera extract capable to alleviate irritation symptoms.

Due to the capability of the new $C$. procera extract to have a protective effect against inflammation and irritation, it had been further elucidated by it combination with Dead Sea water, another regional skin active. Dead Sea minerals are reported in literature for their therapeutic capabilities to treat a variety of skin diseases as well as for their beautifying cosmetic effects [5] [35] [36]. Dead Sea mineral-rich water (DSW; Osmoter ${ }^{\mathrm{TM}}$, a natural commercial composition of Dead Sea water) was combined with $C$. procera extract. In this part of the study the purpose was to test on skin models the effect of a mixture of $C$. procera + DSW extracts versus each active ingredient as stand-alone to search for the involvement of biological pathways that can contribute to skin well-being. The tests 
were carried out on skin equivalents. As a first step, a full micro-array gene expression screening was performed. A deep analysis on transcription level of gene expression could give a preliminary prediction of biological process, which might be involved. Notably, analysis only in gene level is a good starting point, but is not sufficient and additional tests are needed in the protein level. Comparison of the gene profiles of the treatment groups to whole transcriptome GSEA revealed interesting findings, in which biological processes that were significantly enriched, have an opposite direction in the combined treatment group compared to the DSW extract and the $C$. procera extract groups that were treated separately (Figure 4). These biological processes are linked to skin responses to hypoxia, glycolysis and to EMT. The observed processes can contribute to skin maintenance via better coping with stress, optimizing metabolic balance, and regeneration respectively. Therefore, they were further examined by evaluating representative protein/enzymatic biomarkers of relevant detected pathways to strengthen the assumption of their involvement. For each biological process, a representative biomarker was tested. HIF1 enzyme plays a key role in the cell resistance to hypoxia conditions [37]. PFK1 enzyme is crucial for ATP formation as part of glycolysis process [23]. Only treatment with the combination of $C$. procera extract + DSW demonstrated a significant induction of these two enzymes expression compared to each extract solely. Thus, combination of C. procera extract + DSW has surprising effect by their contribution to the processes of energy production and resistance to hypoxia. Together with gene expression results, a patent application had been submitted for the unexpected biological activity of this extracts combination (patent no. $2489269 \mathrm{RIGO} / \mathrm{j}$ ). $C$. procera extract + DSW combination demonstrated also a significant induction in fibronectin enzyme expression. Of note, fibronectin participates in regeneration and healing process and might be related to EMT process [24]. Yet, treatment with $C$. procera extract as stand-alone also enhanced fibronectin expression.

In summary, the combination of $C$. procera extract + DSW is suggested to have superiority by affecting skin in both gene expression level and protein level following topical application. These effects might be in terms of glycolysis and energy production, coping with hypoxia and ECM regeneration.

\section{Conclusion}

The biological effects of $C$. procera callus extract on skin were elucidated in this study, solely and in combination with Dead Sea minerals. C. procera callus extract is a new active ingredient in cosmetic formulae, lately introduced to INCI list of ingredients and is the only cosmetic ingredient extracted of the plant $C$. procera. Study results on laboratory skin models reveal that $C$. procera extract exhibits a protective biological activity against irritation and inflammation at the biochemical level. Furthermore, a combination of $C$. procera extract and DSW extract demonstrates a potential contribution for skin wellbeing via increase energy production, resistance to hypoxia and ECM balance. Taken together, all 
presented results suggest that $C$. procera callus extract might support skin balance and wellbeing at the molecular level. Hence, it is recommended for new cosmetic formulae as standalone or in combination with Dead Sea water, in the effort to achieve anti-aging bio-activity that works on the molecular level beyond skin aging symptoms, especially via skin calming effects and skin energy enhancement.

\section{Acknowledgements}

This study was supported by the Israeli Chief Scientist under project number 55243. The authors thank Mr. Robert Holtz of BioInnovation Laboratories Inc., Lakewood, USA. The authors thank Mr. Ron Michael, AHAVA CEO for his vision to adopt $C$. procera plant as a new active ingredient for cosmetic application.

\section{References}

[1] Spencer, N. (2017) Top Glubal Consumer Trends 2017 Part 2: Authenticity and Personalization.

https://www.cosmeticsdesign-asia.com/Article/2017/02/01/Top-global-consumer-tr ends-2017-authenticity-and-personalisation

[2] Imosemi, I.O. (2016) Evaluation of the Toxicity, Medicinal Use and Pharmacological Actions of Calotropis Procera. EJPMR, 3, 28-39.

[3] Yaniv, Z. and Koltai, H. (2018) Calotrtopis procera, Apple of Sodom. Ethnobotanical Review and Medicinal Activities. Israel Journal of Plant Sciences, Accepted for Publication.

[4] Parrotta, J.A. (2001) Healing Plants of Peninsular India. CAB International, Wallingford, UK and New York, 944. https://doi.org/10.1079/9780851995014.0000

[5] Moses, S.W., et al. (2006) The Dead Sea, a Unique Natural Health Resort. The Israel Medical Association Journal, 8, 483-488.

[6] Oron, M., Portugal-Cohen, M., Horev, L., Tauber, G., Cohen, D., David, M., Ma'or, Z., Ben-Yehuda Greenwald, M., Cohen, D., Kohen, R., Hodak, E. and Pavlovsky, L. (2017) Complementary Non-Invasive Methods to Assess Disease State in Psoriasis Vulgaris Patients. Clinical \& Experimental Dermatology and Therapies, 2017, $1-10$.

[7] Portugal-Cohen, M., Miriam, O., Merrik, E., Ma’or, Z., Ben-Amitai, D., Yogev, H. and Zvulunov, A. (2011) A Dead Sea Water-Enriched Body Cream Improves Skin Severity Scores in Children with Atopic Dermatitis. Journal of Cosmetics, Dermatological Sciences and Applications, 1, 71-78.

https://doi.org/10.4236/jcdsa.2011.13012

[8] Portugal-Cohen, M., Dominguez, F.M., Oron, M., Holtz, R. and Ma'or, Z. (2015) Dead Sea Minerals-Induced Positive Stress as an Innovative Resource for Skincare Actives. Journal of Cosmetics, Dermatological Sciences and Applications, 5, 22-35. https://doi.org/10.4236/jcdsa.2015.51004

[9] Portugal-Cohen, M., et al. (2017) Antipollution Skin Protection-A New Paradigm and Its Demonstration on Two Active Compounds. Clinical, Cosmetic and Investigational Dermatology, 10, 185-193. https://doi.org/10.2147/CCID.S129437

[10] Portugal-Cohen, M., et al. (2009) Protective Effects of a Cream Containing Dead 
Sea Minerals against UVB-Induced Stress in Human Skin. Experimental Dermatology, 18, 781-788. https://doi.org/10.1111/j.1600-0625.2009.00865.x

[11] Ma'or, Z., Meshulam-Simon, G., Yehuda, S. and Gavrieli, J.A. (1999) Antiwrinkle and Skin Moisturizing Effects of a Mineral-Algal-Botanical Complex. Journal of Cosmetic Science, 51, 27-36.

[12] Wineman, E., et al. (2012) Photo-Damage Protective Effect of Two Facial Products, Containing a Unique Complex of Dead Sea Minerals and Himalayan Actives. Journal of Cosmetic Dermatology, 11, 183-192.

[13] Parliament, E.N.o.t.E., Regulation (EC) No 1223/2009 of the European Parliament and of the Council of 30 November 2009 on Cosmetic Products 2009.

[14] Datta, H.S. and Paramesh, R. (2010) Trends in Aging and Skin Care: Ayurvedic Concepts. Journal of Ayurveda and Integrative Medicine, 1, 110-113. https://doi.org/10.4103/0975-9476.65081

[15] Uitto, J. (1997) Understanding Premature Skin Aging. The New England Journal of Medicine, 337, 1463-1465. https://doi.org/10.1056/NEJM199711133372011

[16] Hashizume, H. (2004) Skin Aging and Dry Skin. The Journal of Dermatology, 31, 603-609. https://doi.org/10.1111/j.1346-8138.2004.tb00565.x

[17] Helfrich, Y.R., Sachs, D.L. and Voorhees, J.J. (2008) Overview of Skin Aging and Photoaging. Dermatology Nursing, 20, 177-183.

[18] Hoeijmakers, J.H. (2009) DNA Damage, Aging, and Cancer. The New England Journal of Medicine, 361, 1475-1485. https://doi.org/10.1056/NEJMra0804615

[19] Portugal-Cohen, M., et al. (2011) Skin Organ Culture as a Model to Study Oxidative Stress, Inflammation and Structural Alterations Associated with UVB-Induced Photodamage. Experimental Dermatology, 20, 749-755.

https://doi.org/10.1111/j.1600-0625.2011.01317.x

[20] Murashige, T. and Skoog, F. (1962) Revised Medium for Rapid Growth and Bioassays with Tobacco Tissue Cultures. Physiologia Plantarum, 15, 473-497. https://doi.org/10.1111/j.1399-3054.1962.tb08052.x

[21] Tripathi, P.K., Awasthi, S., Kanojiya, S., Tripathi, V. and Mishra, D.K. (2013) Callus Culture and in Vitro Biosynthesis of Cardiac Glycosides from Calotropis gigantea in Vitro Cellular \& Developmental Biology. Plant, 49, 455-460.

[22] Manresa, M.C. and Taylor, C.T. (2017) Hypoxia Inducible Factor (HIF) Hydroxylases as Regulators of Intestinal Epithelial Barrier Function. Cellular and Molecular Gastroenterology and Hepatology, 3, 303-315. https://doi.org/10.1016/j.jcmgh.2017.02.004

[23] Mor, I., Cheung, E.C. and Vousden, K.H. (2011) Control of Glycolysis through Regulation of PFK1: Old Friends and Recent Additions. Cold Spring Harbor Symposia on Quantitative Biology, 76, 211-216. https://doi.org/10.1101/sqb.2011.76.010868

[24] Stone, R.C., et al. (2016) Epithelial-Mesenchymal Transition in Tissue Repair and Fibrosis. Cell and Tissue Research, 365, 495-506. https://doi.org/10.1007/s00441-016-2464-0

[25] Fisher, G.J., et al. (2002) Mechanisms of Photoaging and Chronological Skin Aging. Archives of Dermatology, 138, 1462-1470. https://doi.org/10.1001/archderm.138.11.1462

[26] Pillai, S., Oresajo, C. and Hayward, J. (2005) Ultraviolet Radiation and Skin Aging: Roles of Reactive Oxygen Species, Inflammation and Protease Activation, and Strategies for Prevention of Inflammation-Induced Matrix Degradation-A Review. 
International Journal of Cosmetic Science, 27, 17-34. https://doi.org/10.1111/j.1467-2494.2004.00241.x

[27] Belz, R.G. and Duke, S.O. (2014) Herbicides and Plant Hormesis. Pest Management Science, 70, 698-707. https://doi.org/10.1002/ps.3726

[28] Mattson, M.P. (2008) Hormesis Defined. Ageing Research Reviews, 7, 1-7. https://doi.org/10.1016/j.arr.2007.08.007

[29] Desai, A., et al. (2009) META060 Inhibits Multiple Kinases in the NF-kappaB Pathway and Suppresses LPS-Mediated Inflammation in Vitro and ex Vivo. Inflammation Research, 58, 229-234. https://doi.org/10.1007/s00011-008-8162-y

[30] Le, T.K., et al. (1997) Effect of a Topical Corticosteroid, a Retinoid and a Vitamin D3 Derivative on Sodium Dodecyl Sulphate Induced Skin Irritation. Contact Dermatitis, 37, 19-26. https://doi.org/10.1111/j.1600-0536.1997.tb00369.x

[31] Matsukawa, A., et al. (1997) Analysis of the Inflammatory Cytokine Network among TNF Alpha, IL-1 Beta, IL-1 Receptor Antagonist, and IL-8 in LPS-Induced Rabbit Arthritis. Laboratory Investigation, 76, 629-638.

[32] Reimann, T., et al. (1994) Lipopolysaccharide Induces Activation of the Raf-1/MAP Kinase Pathway. A Putative Role for Raf-1 in the Induction of the IL-1 Beta and the TNF-Alpha Genes. The Journal of Immunology, 153, 5740-5749.

[33] Corsini, E. and Galli, C.L. (1998) Cytokines and Irritant Contact Dermatitis. Toxicology Letters, 102-103, 277-282.

[34] Gibbs, S. (2009) In Vitro Irritation Models and Immune Reactions. Skin Pharmacology and Physiology, 22, 103-113. https://doi.org/10.1159/000178869

[35] Halevy, S. and Sukenik, S. (1998) Different Modalities of Spa Therapy for Skin Diseases at the Dead Sea Area. Archives of Dermatology, 134, 1416-1420.

https://doi.org/10.1001/archderm.134.11.1416

[36] Ma'or, Z., Yehuda, S. and Voss, W. (1997) Skin Smoothing Effects of Dead Sea Minerals: Comparative Profilometric Evaluation of Skin Surface. International Journal of Cosmetic Science, 19, 105-110. https://doi.org/10.1111/j.1467-2494.1997.tb00173.x

[37] Wynn, T.A. (2008) Cellular and Molecular Mechanisms of Fibrosis. The Journal of Pathology, 214, 199-210. https://doi.org/10.1002/path.2277 